OPEN ACCESS

Edited by:

Bing Du,

East China Normal University, China

Reviewed by:

Paulraj Kanmani,

Tohoku University, Japan

Imke Steffen,

University of Veterinary Medicine

Hannover, Germany

*Correspondence:

Weilin Chen

cwl@szu.edu.cn

Specialty section: This article was submitted to Viral Immunology,

a section of the journal

Frontiers in Immunology

Received: 16 November 2021 Accepted: 07 February 2022

Published: 02 March 2022

Citation:

Xiao Y, Chen X, Wang Z, Quan J, Zhao X, Tang H, Wu H, Di Q, Wu Z and

Chen W (2022) Succinate Is a Natural Suppressor of Antiviral Immune Response by Targeting MAVS.

Front. Immunol. 13:816378. doi: 10.3389/fimmu.2022.816378

\section{Succinate Is a Natural Suppressor of Antiviral Immune Response by Targeting MAVS}

\author{
Yue Xiao ${ }^{1}$, Xinyi Chen ${ }^{1}$, Zhun Wang ${ }^{2}$, Jiazheng Quan ${ }^{1}$, Xibao Zhao ${ }^{1}$, Haimei Tang ${ }^{1}$, \\ Han $W u^{1}$, Qianqian $D i^{1}$, Zherui $W u^{1}$ and Weilin Chen ${ }^{1 *}$ \\ 1 Department of Immunology, School of Medicine, Shenzhen University, Shenzhen, China, ${ }^{2}$ Technological Center, \\ Changchun Customs, Changchun, China
}

Succinate is at the crossroads of multiple metabolic pathways and plays a role in several immune responses acting as an inflammation signal. However, whether succinate regulates antiviral immune response remains unclear. Here, we found that the production of succinate was reduced in RAW264.7 cells during vesicular stomatitis virus (VSV) infection. Using diethyl succinate to pretreat the mouse peritoneal macrophages and RAW264.7 cells before VSV infection, the production of interferon- $\beta$ (IFN- $\beta$ ), chemokine (C-X-C motif) ligand 10 (CXCL-10), and IFN-stimulated genes 15 (ISG15) was significantly decreased, following which the VSV replication in diethyl succinate-pretreated cells was obviously increased. Moreover, succinate decreased the expression of IFN- $\beta$ in serum, lung, and spleen derived from the VSV-infected mice. The overall survival rate in the VSV-infected mice with diethyl succinate pretreatment was also remarkably downregulated. Furthermore, we identified that succinate inhibited the activation of MAVS-TBK1-IRF3 signaling by suppressing the formation of MAVS aggregates. Our findings provide previously unrecognized roles of succinate in antiviral immune response and establish a novel link between metabolism and innate immune response.

Keywords: succinate, metabolism, VSV, antiviral immune response, MAVS

\section{INTRODUCTION}

Cellular metabolism is now considered a prominent route in our quest to understand immune responses (1). In recent years, the Krebs cycle metabolite succinate has been in succession reported that concerned with immune responses. Succinate could induce migratory responses of dendritic cells and act in synergy with Toll-like receptor ligands for proinflammatory cytokine production

\footnotetext{
Abbreviations: GPR91, G protein-coupled receptor; LPS, lipopolysaccharide; IFNs, type I interferons; PRRs, pattern recognition receptors; RIG-I, retinoic-acid inducible gene I; RLRs, retinoic-acid inducible gene I (RIG-I)-like receptors; MDA5, melanoma differentiation-associated gene 5; IRF3, interferon regulatory factor 3; HBP, hexosamine biosynthesis pathway; O-GlcNAc, $O$-linked $\beta$-N-acetylglucosamine; SAMHD1, histidine/aspartic acid domain-containing protein 1; MPM, mouse peritoneal macrophages; VSV, vesicular stomatitis virus; GLUT, glucose transporter; TCA, tricarboxylic acid; IDH1, isocitrate dehydrogenase 1; OGDH, $\alpha$-ketoglutarate dehydrogenase; OXPHOS, oxidative phosphorylation; Ds, diethyl succinate; MOI, multiplicity of infection; CXCL-10, chemokine (C-X-C motif) ligand 10; ISG15, IFN-stimulated genes 15; PG, prostaglandin; iNSCs, induced neural stem cells.
} 
through binding to succinate receptor SUNCR1, also known as G protein-coupled receptor (GPR91) (2). SUNCR1 is expressed on various types of cells including immune cells (3). Via the succinate-SUNCR1 signaling axis, succinate triggered proinflammatory response in macrophages during antigeninduced arthritis and induced a type 2 immune response in the small intestine (4-6). In the lipopolysaccharide (LPS)-stimulated macrophages, succinate acted as a metabolite in innate immune signaling to enhance interleukin-1 $\beta$ production through hypoxia-inducible factor- $1 \alpha$ (7). However, whether succinate has a role in antiviral immune response remains unclear.

Type I interferons (IFNs) produced by nearly all cell types play a pivotal role in host defense against viral infection. Type I IFNs, including IFN- $\alpha$, IFN- $\beta$, and other IFN family members, are induced by the activation of cell-surface or intracellular pattern recognition receptors (PRRs) (8). During RNA viral infection, cytoplasmic RNA species are recognized by retinoicacid inducible gene I (RIG-I)-like receptors (RLRs, a member of PRRs) composed of RIG-I and melanoma differentiationassociated gene 5 (MDA5). RIG-I or MDA5 detects and binds with viral RNA via their C-terminal repressor (9). Upon sensing RNA, the activation of RIG-I and MDA5 undergoes a conformational change to recruit mitochondrial antiviral signaling (MAVS) protein. The C-terminus transmembrane domain of MAVS is necessary for its localization in the mitochondrial outer membrane. Once activated, MAVS develops a functional prion-like structure at the mitochondria and activates the downstream protein kinase TBK1, resulting in phosphorylation of the transcription factor interferon regulatory factor 3 (IRF3) and its nuclear translocation to drive the production of type I IFN (8-11). Intracellular RIG-I-MAVS signaling and type I IFN production are orchestrated by numerous mechanisms (12-14). Recently, energy metabolism in the regulation of antiviral innate immunity is becoming the new research field to understand the host antiviral immune response. Zhang et al. demonstrated that glucose-generated lactate acts as a regulator of MAVS to inhibit RLR signaling, allowing a cross-regulation between antiviral signaling and energy metabolism (15). The study, which showed that hexosamine biosynthesis pathway (HBP)-associated $O$-linked $\beta$-N-acetylglucosamine ( $O$-GlcNAc) mediated the $\mathrm{O}$ GlcNacylation and stabilization of sterile alpha motif and histidine/aspartic acid domain-containing protein 1 (SAMHD1) to positively regulate host antiviral response against $\mathrm{HBV}$, revealed a link between $\mathrm{HBP}$ and innate antiviral immunity further (16). Therefore, it is necessary to explore more biochemical regulations between energy metabolism and antiviral response for creating exciting novel opportunities in therapeutic applications for virus infection in future.

In this study, using the mouse peritoneal macrophages and RAW264.7 cell culture systems, as well as a VSV-infection mouse model, we found that succinate negatively regulates the innate immunity in response to VSV infection in vitro and in vivo. Our results revealed that succinate could inhibit aggregation of MAVS and its activation. These findings suggest a vital role of succinate in limiting RLR signaling through targeting MAVS.

\section{MATERIALS AND METHODS}

\section{Mice and Cell Culture}

C57BL/6 mice (female, 6- to 8-week-old) were purchased from the Guangdong Medical Laboratory Animal Center (Guangdong, China) and housed in SPF breeding units. The animal experimental manipulation was performed according to the National Institute of Health Guide for the Care and Use of Laboratory Animals with the approval of Shenzhen University (Approval Number: AEWC-20200316).

Mouse peritoneal macrophages (MPMs) were harvested from mice which had received a $2-\mathrm{ml}$ intraperitoneal injection of $3 \%$ Brewer thioglycollate broth (Sigma, Poole, UK) 3 days prior to collection. The MPMs were cultured in RPMI 1640 with $10 \%$ FCS. Mouse macrophage cell line RAW264.7 cells and HEK293T cells were purchased from ATCC and cultured in DMEM medium containing $10 \%$ FBS.

\section{Plasmids, Antibodies, and Reagents}

Myc-tagged RIG-I (N), Myc-tagged MAVS, Myc-tagged TBK1, and Myc-tagged IRF3-5D plasmids were constructed by cloning into the pcDNA3.1 vector. Antibodies against RIG-I (\#3743), MAVS (\#83000), TBK1 (\#3504), IRF3 (\#4302), Phospho-TBK1 (5483), and Phospho-IRF3 (\#4947) were purchased from Cell Signaling Technology (Danvers, MA, USA). Succinic acid (\#S9512) was purchased from Sigma-Aldrich (St. Louis, USA).

\section{Virus Infection and Reagent Treatment}

Cells were infected with VSV (multiplicity of infection (MOI) = 1 ) or VSV-eGFP (MOI $=0.01$ ) for the indicated hours. For in vivo infection, the regular dose of VSV for mice was $1 \times 10^{8} \mathrm{PFU} /$ $\mathrm{g}$ by intraperitoneal injection and the half-lethal dose was $1.3 \times$ $10^{7} \mathrm{PFU} / \mathrm{g}$ by intravenous injection. The doses of dimethyl succinate (Ds) in cells and mice were 5 and $62.5 \mathrm{mM}$ in $100 \mu \mathrm{l}$ PBS per mouse separately.

\section{Cell Transfection and Dual-Luciferase Reporter Assay}

Plasmids were transfected into HEK293T cells using jetPRIME transfection reagents according to the manufacturer's protocol. Dual-luciferase reporter assay was performed in a 96-well plate with 90-ng luciferase reporter plasmids (IFN- $\beta$ or ISRE luciferase reporter), $10 \mathrm{ng}$ indicated Renilla luciferase construct phRL-TK (Promega, for normalizing the transfection efficiency), and $50 \mathrm{ng}$ indicated expressing plasmids. The cells were harvested at $24 \mathrm{~h}$ post transfection. Luciferase assays were performed using a dualspecific luciferase assay kit (\#E1960, Promega, Madison, WI, USA) in accordance with instructions. All samples were set as triplicate, and the folds were calculated relative to the baseline control in each experiment.

\section{Real-Time Quantitative PCR}

The total RNA of cells was isolated by TRIzol reagent (Takara, Japan). Reverse transcription was used to generate cDNAs from RNA by Reverse Transcriptase M-MLV (RNase H-) (Takara, Shiga, Japan). Real-time quantitative PCR was performed with 
Hieff $^{\mathrm{TM}}$ qPCR SYBR Green Master Mix (Yeasen, Shanghai, China) and detected with the Analytik Jena qTOWER ${ }^{3}$ PCR system (Jena, Germany). Data were determined by normalization of expression of $\beta$-actin in each sample and analyzed using the 2$\Delta \Delta \mathrm{CT}$ method. Gene-specific primer sequences were as the following: mouse $\beta$-actin, 5'-AGTGTGACGTTGACATCCGT$3^{\prime}$ and $5^{\prime}$-GCAGCTCAGTAACAGTCCGC-3' ${ }^{\prime}$; mouse IFN- $\beta$, $5^{\prime}$ ATGAGTGGTGGTTGCAGGC- $3^{\prime}$ and $5^{\prime}$-TGACCTTTCAAA TGCAGTAGATTCA-3'; mouse GLUT1, 5' -TCATTGTCGGC CTCCTCATT-3' and 5'-TAGGGTGGCAGAACTTGAGG-3'; mouse GLUT4, 5'-GCCATCGTCATTGGCATTCT-3' and 5'CTCCAGGTTCCGGATGATGT-3'; mouse IDH1, 5'-ACTCA GTCGCCCAAGGTTAT-3' and 5'-GTAGTGACGTGTG ACAGTGC-3'; mouse OGDH, 5'-CCACCCTGAGGCAA GAACTA- $3^{\prime}$ and 5'-CCTTCCCAGTGCAGAAGAGA-3'.

\section{Western Blotting Assay}

Cell lysates were prepared using RIPA buffer containing a protease inhibitor cocktail (Sigma-Aldrich, St. Louis, USA) and boiled with $1 \%$ SDS sample buffer. 20 to $40 \mu \mathrm{g}$ of proteins was separated using $10 \%$ SDA-PAGE gels and then electro-transferred to nitrocellulose filter membranes (Millipore, Bedford, MA. USA). The membranes were blocked in $5 \%$ non-fat milk and immunoblotted with primary specific antibodies in concentrations as recommended by the manufacturer for overnight at $4^{\circ} \mathrm{C}$. Secondary antibodies were conjugated with HRP. Blots were scanned using the Tanon 6100B Imaging System.

\section{Enzyme-Linked Immunosorbent Assay}

RAW264.7 and peritoneal macrophage culture supernatants and mouse serum were collected and stored at $-80^{\circ} \mathrm{C}$. IFN- $\beta$ was measured using a Mouse IFN- $\beta$ ELISA Kit (BioLegend, San Diego, CA, USA) according to the manufacturer's protocol.

\section{Flow Cytometric Assay}

Cells were infected with VSV-eGFP or PBS for $16 \mathrm{~h}$ and underwent pancreatin digestion following twice cold PBS washing. Total cells were analyzed using Beckman CytoFLEX S. Results were analyzed by FlowJo version 10.4 software.

\section{Hematoxykin and Eosin Staining}

Lungs from control or VSV-infected mice were fixed in $4 \%$ (weight/vol.) paraformaldehyde, embedded into paraffin, sectioned, stained with hematoxylin and eosin solution, and observed under the microscope.

\section{Semi-Denaturing Detergent Agarose Gel Electrophoresis}

Semi-denaturing detergent agarose gel electrophoresis (SDDAGE) was performed according to a published protocol with minor modifications (11) Briefly, equal amounts of the wholecell lysate were resuspended in one-third volume of $4 \times$ sample buffer $(2 \times$ TAE, $40 \%$ glycerol, $8 \%$ SDS, and $0.02 \%$ bromophenol blue). Samples were incubated for $5 \mathrm{~min}$ at room temperature and loaded onto a vertical $1.5 \%$ agarose gel containing a final concentration of $0.1 \%$ SDS. After electrophoresis in the running buffer $(1 \times$ TBE and $0.1 \%$ SDS $)$ for $40 \mathrm{~min}$ with a constant voltage of $100 \mathrm{~V}$ at $4^{\circ} \mathrm{C}$, proteins were transferred to PVDF membranes using a liquid transfer system in preparation for Western blotting analysis.

\section{Cellular Succinate Measurement}

Intracellular succinate levels were measured using a Succinate Assay Kit (\#ab204718, Abcam, Cambridge, USA) according to the manufacturer's protocol. Briefly, $2 \times 10^{6}$ cells/each assay were harvested and washed with cold PBS. Cells were resuspended in succinate assay buffer and homogenized quickly. Any insoluble material was removed by centrifugation. The collected supernatant was further deproteinized using a $10-\mathrm{kDa}$ spin column (\#ab93349, Abcam, Cambridge, USA) and subjected to succinate measurement.

\section{Statistical Analysis}

All experiments assays were conducted at least three times with samples in triplicates. Statistical analysis comparisons between groups were determined by unpaired two-tailed Student's $t$-test. $\mathrm{p}$ values $<0.05$ were considered statistically significant. Animal survival curves were estimated for each group, the Kaplan-Meier method was adopted to generate graphs, and the survival curves were analyzed by log-rank analysis. GraphPad Prism 7.0 software (GraphPad Software, San Diego, CA) was used for all statistical analyses.

\section{RESULTS}

\section{VSV Infection Promotes Glucose Metabolism in Macrophages}

To test glucose metabolic changes in response to viral challenge, we challenged RAW264.7 cells and mouse peritoneal macrophages (MPM) with vesicular stomatitis virus (VSV) or PBS as normal control and detected the expression levels of key markers in the glucose metabolic. RT-qPCR analysis showed that the mRNA levels of glucose transporter (GLUT) 1 and GLUT4 were significantly increased in the RAW264.7 cells and MPM infected with VSV for $12 \mathrm{~h}$, while there were no changes in the PBS-treated group (Figures 1A, B). The mRNA levels of tricarboxylic acid (TCA) cycle key enzymes isocitrate dehydrogenase 1 (IDH1) and $\alpha$-ketoglutarate dehydrogenase (OGDH) in RAW264.7 cells and MPM were gradually decreased with the prolongation of VSV infection time (Figures 1C, D). Besides, the Succinate Assay Kit (Colorimetric) was used to detect the TCA cycle intermediate succinate levels in the RAW264.7 cells; the results showed that succinate levels were also decreased in response to VSV infection (Figures 1E, F). These findings demonstrated that VSV infection could shift energy metabolism from oxidative phosphorylation (OXPHOS) to glycolysis in mouse macrophages.

\section{Succinate Negatively Regulates Virus-Induced IFN- $\beta$ Production}

To explore the role of succinate in antiviral response, we used VSV to examine the effect of succinate on the production of IFN- $\beta$. 
RAW264.7

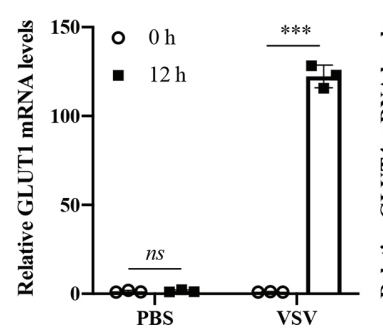

C

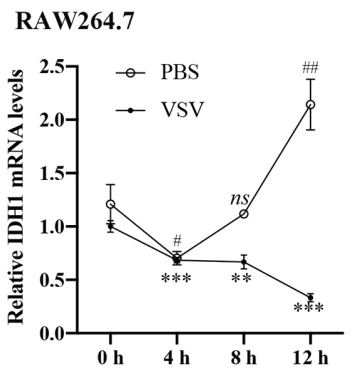

RAW264.7

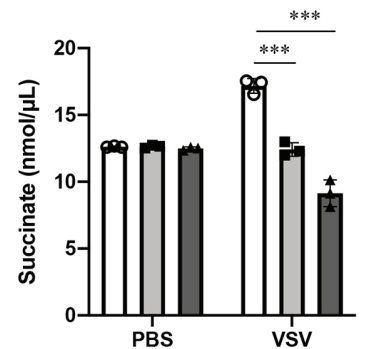

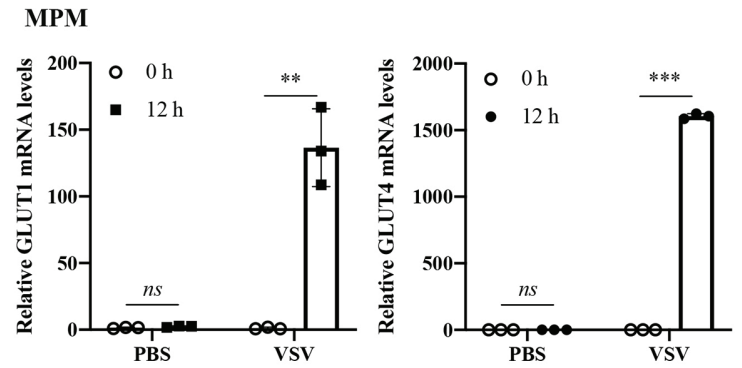

D

MPM
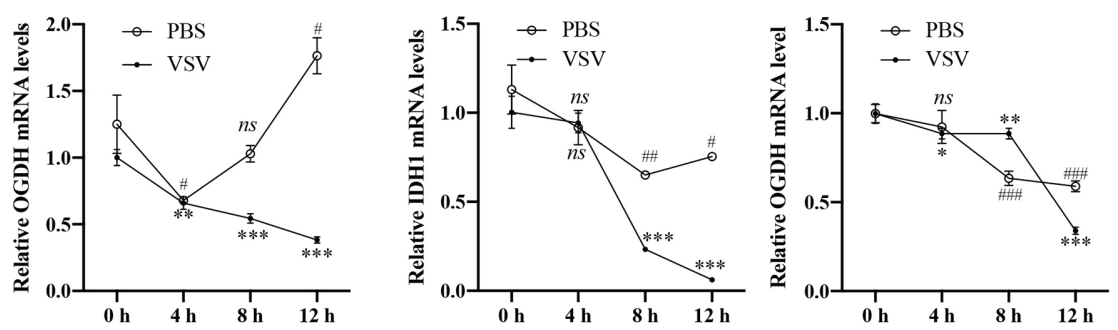

MPM

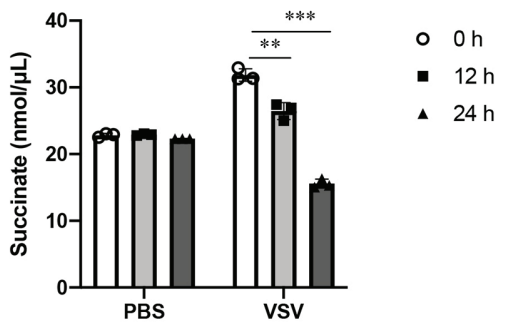

FIGURE 1 | VSV infection promotes glucose metabolism in macrophages. (A, B) The mRNA levels of GLUT1 and GLUT4 were determined by RT-qPCR in RAW264.7 cells (A) and MPM (B) infected with vesicular stomatitis virus (VSV) (multiplicity of infection (MOI) =1) or PBS for $12 \mathrm{~h}$. (C, D) The mRNA levels of IDH1 (C) and OGDH (D) were determined by RT-qPCR in RAW264.7 cells $(\mathbf{C})$ and MPM (D) infected with VSV (MOI =1) or PBS for 4, 8, or $12 \mathrm{~h}$. (E, F) Succinate abundance in RAW264.7 cells $(\mathbf{E})$ and MPM (F) infected with VSV $(\mathrm{MOI}=1)$ or PBS for 12 and $24 \mathrm{~h}$. All data are represented as means \pm SD. Significance calculated using the unpaired t-test ( ${ }^{*} v s$. $0 \mathrm{~h}$

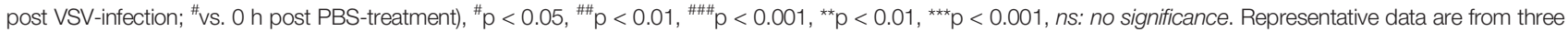
independent experiments.

RAW264.7 cells or MPM were pretreated with or without $5 \mathrm{mM}$ cellpermeable diethyl succinate (Ds) for $3 \mathrm{~h}$ and then infected with VSV at multiplicity of infection $(\mathrm{MOI})=1$ for $0,4,8$, or $24 \mathrm{~h}$. The cells treated with PBS were used as control group. The mRNA levels of IFN- $\beta$ were lower in Ds-pretreated RAW264.7 cells than those in DMSO-pretreated cells (Figure 2A). Enzyme-linked immunosorbent assay (ELISA) showed that the protein level of IFN- $\beta$ decreased by about 75\% in the media of VSV-infected RAW264.7 cells cultured for $24 \mathrm{~h}$ in the presence of Ds (Figure 2B). Furthermore, in the Dspretreated MPM, the mRNA levels of IFN- $\beta$ were significantly lower than those in the DMSO-pretreated MPM (Figure 2C). ELISA showed that IFN- $\beta$ production decreased by about $43 \%$ in the media of VSV-infected MPM cultured for $24 \mathrm{~h}$ in the presence of Ds (Figure 2D). These results indicate that succinate inhibits the production of IFN- $\beta$ in macrophages infected with VSV.

\section{Succinate Inhibits Cellular Antiviral Response in Macrophages}

To investigate whether succinate affected cell antiviral ability, we treated RAW264.7 cells and MPM with Ds/DMSO before being infected with VSV. VSV replication and titer in cells were measured. As shown in Figure 3A, the mRNA levels of VSV in the Ds-pretreated cells with VSV infection for $12 \mathrm{~h}$ were higher than those in control cells. TCID50 assay showed that higher VSV titers in Ds-treated cells were detected in the supernatants of RAW264.7 cells and MPM with VSV infection for 12 and $24 \mathrm{~h}$ (Figure 3B). Furthermore, flow cytometry was used to confirm the role of succinate in VSV replication. The results revealed that $26.7 \%$ cells were infected (GFP+) in cells pretreated with Ds, compared to $17.5 \%$ of GFP-positive cells in DMSO-pretreated cells at $16 \mathrm{~h}$ post VSV-eGFP infection in 


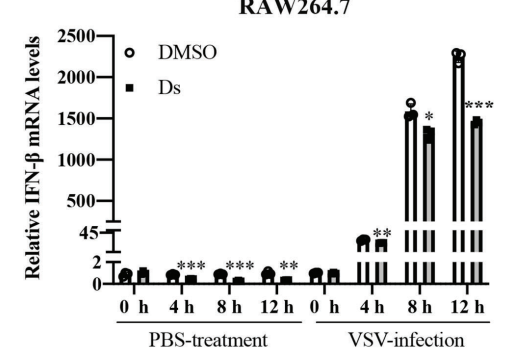

RAW264.7

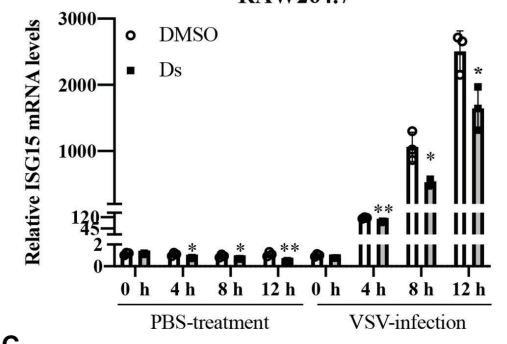

C
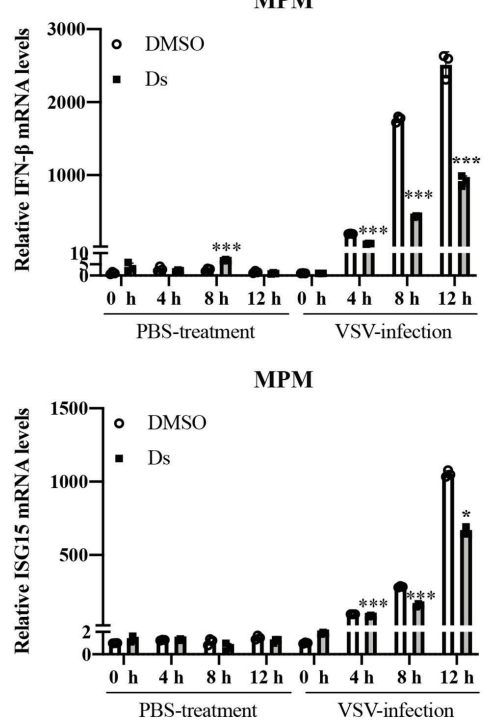

RAW264.7

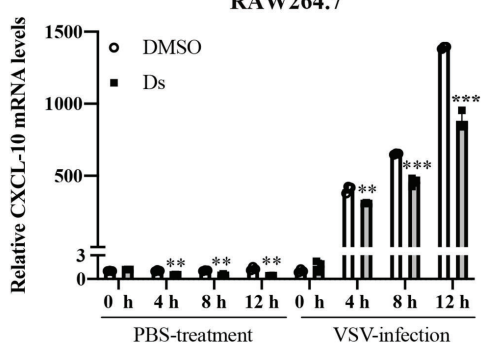

B

RAW264.7

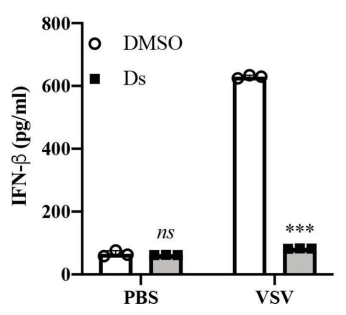

MPM
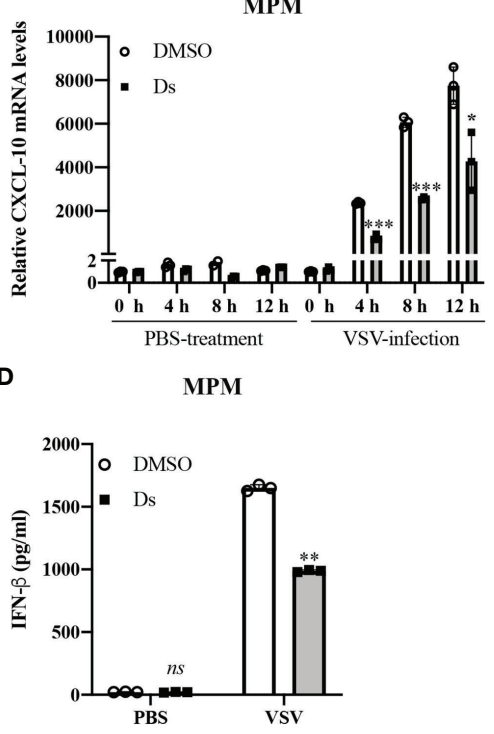

FIGURE 2 | Succinate negatively regulates virus-induced IFN- $\beta$ production. (A, B) RAW264.7 cells were pretreated with or without 5 mM diethyl succinate (Ds) for 3 $\mathrm{h}$ and then infected with VSV (MOI = 1) or PBS for 0, 4, 8, or $12 \mathrm{~h}$. (A) The mRNA levels of IFN- $\beta$, CXCL-10, and ISG15 were measured by RT-qPCR in RAW264.7 cells. (B) The protein levels of IFN- $\beta$ were measured by ELISA in RAW264.7 cells. (C, D) Mouse peritoneal macrophages (MPM) were pretreated with or without 5 $\mathrm{mM}$ diethyl succinate (Ds) for $3 \mathrm{~h}$ and then infected with VSV (MOI = 1) for 0, 4, 8, or 12 h. (C) The mRNA levels of IFN- $\beta$, CXCL-10, and ISG15 were measured by RT-qPCR in MPM cells. (D) The protein levels of IFN- $\beta$ were measured by ELISA in MPM cells. All data are represented as means \pm SD. Significance calculated using the unpaired $t$-test. ${ }^{\star} p<0.05,{ }^{\star \star} p<0.01,{ }^{\star \star *} p<0.001$. Representative data are from three independent experiments.

RAW264.7 cells (Figure 3C left). There were 20.5\% of GFPpositive cells in Ds-pretreated cells, compared to $10.5 \%$ of GFP-positive cells in DMSO-pretreated cells at $16 \mathrm{~h}$ post VSVeGFP infection in MPM (Figure 3C right). Correspondingly, a fluorescence microscope was used to observe the positive cells infected by VSV-eGFP. The results showed that the RAW264.7 cells and MPM pretreated with Ds compared with control cells had more GFP-positive cells (Figure 3D). These data suggest that succinate inhibits cellular antiviral response.

\section{Succinate Suppressed the Antiviral Response of Mice In Vivo}

To prove that succinate affected host antiviral innate response, we challenged mice with VSV $\left(1 \times 10^{8} \mathrm{PFU} / \mathrm{g}\right)(17)$ or PBS by intraperitoneal (i.p.) injection after Ds or PBS injection (i.p.) for $3 \mathrm{~h}$. At $12 \mathrm{~h}$ post VSV infection, the serum, lungs, and spleens of mice were collected to test the IFN- $\beta$ production and VSV loads. The results showed that the production of IFN- $\beta$ in the serum from PBS-injected mice was nearly two-fold higher than in the 
A

RAW264.7

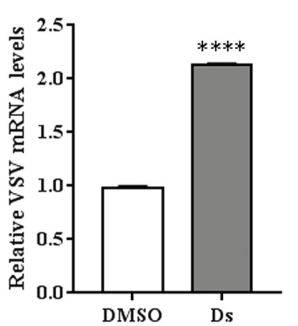

MPM

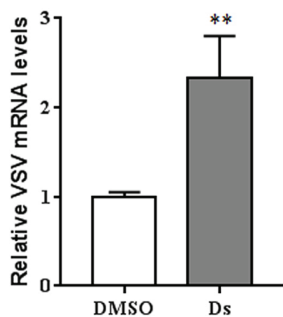

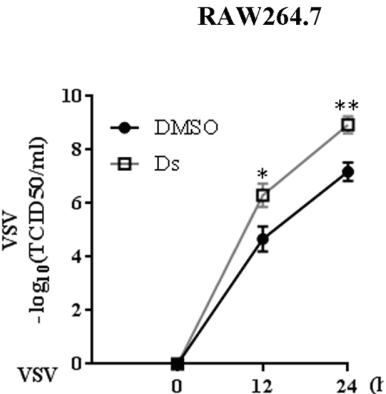

MPM

C
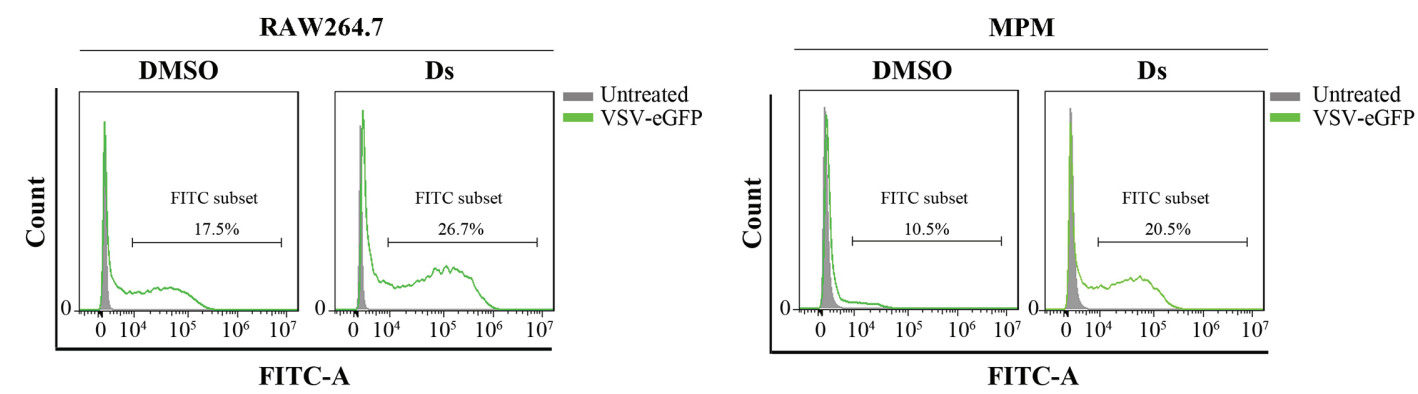

D
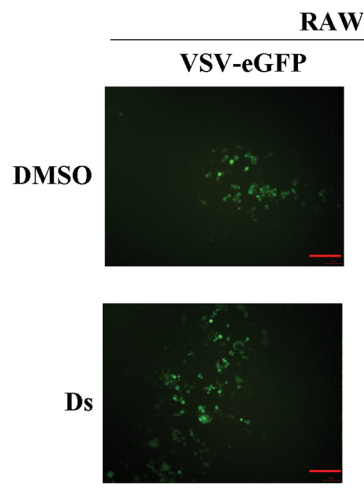

RAW264.7
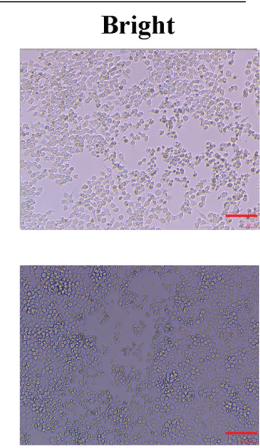

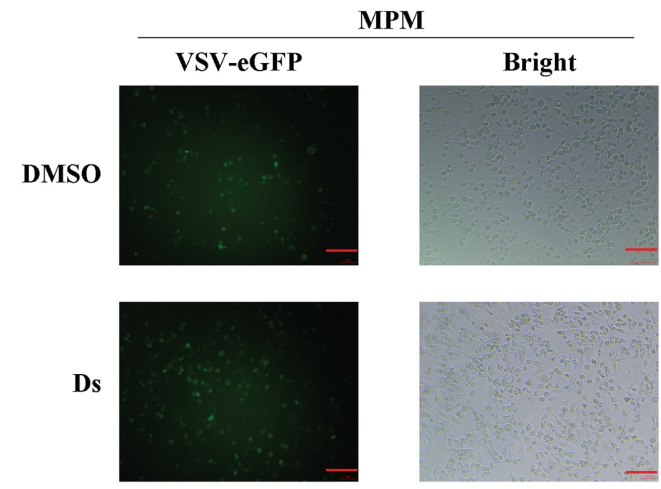

FIGURE 3 | Succinate inhibits VSV replication in macrophages. RAW264.7 cells and MPM were pretreated with $5 \mathrm{mM}$ Ds or DMSO for $3 \mathrm{~h}$ followed infection by VSV. (A) The mRNA levels of VSV were determined by RT-qPCR in RAW264.7 cells and MPM infected with VSV $(M O I=1)$ for $12 \mathrm{~h}$. Data are represented as means \pm SD. Significance calculated using the unpaired $t$-test. ${ }^{\star \star} p<0.01,{ }^{\star \star \star \star} p<0.0001$. (B) VSV titers were determined by TCID50 assay in the supernatants of RAW264.7 cells and MPM with VSV $(\mathrm{MOI}=1)$ challenged for 12 and $24 \mathrm{~h}$. Data are represented as means \pm SD. Significance calculated using the unpaired $t$-test. ${ }^{*} p<0.05$, ${ }^{* *} p<$ 0.01. (C) The percentage of positive VSV-infected cells were determined by flow cytometry in RAW264.7 cells and MPM challenged with VSV-eGFP (MOI $=0.01$ ) for $16 \mathrm{~h}$. (D) RAW264.7 cells and MPM infected with VSV-eGFP $(\mathrm{MOI}=0.01)$ that underwent fluorescence microscopy for $16 \mathrm{~h}$, red scale bar $=100 \mu \mathrm{m}$. Representative data or images are from three independent experiments.

serum from Ds-injected mice in the VSV-infected group (Figure 4A). The IFN- $\beta$ mRNA levels of lungs and spleens from the Ds-injected mice were markedly reduced compared to PBS-injected mice in the VSV-infected group (Figure 4B). VSV titers in the lungs and spleens from the Ds-injected mice were lower than that in the PBS-injected mice (Figure 4C). H\&E staining showed that the infiltration of inflammatory cells in the lung sections of PBS-injected mice was fewer than that in the Dsinjected mice after infection with VSV (Figure 4D). Furthermore, we studied the effects of succinate on the survival of VSV-infected mice. The Ds or PBS-pretreated mice $(n=8 /$ group) were challenged with a half-lethal dose of VSV $\left(1.3 \times 10^{7}\right.$ $\mathrm{PFU} / \mathrm{g}$ ) by intravenous (i.v.) injection, and the times to death of the mice were recorded. As shown in Figure 4E, at $20 \mathrm{~h}$ post VSV infection, all the mice were dead in the Ds-treated group; meanwhile, there were six mice still alive in the PBS-treated group. The survival assay showed that mice treated with succinate were more susceptible to VSV infection than control mice. Taken together, these findings demonstrate that succinate suppresses the antiviral response of mice. 


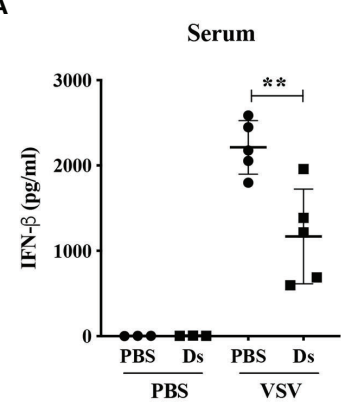

C

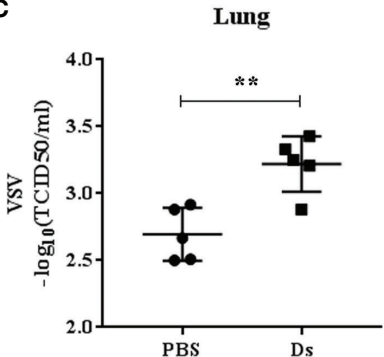

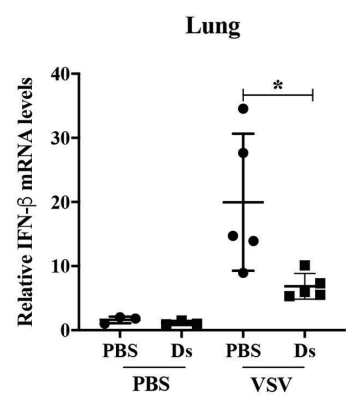

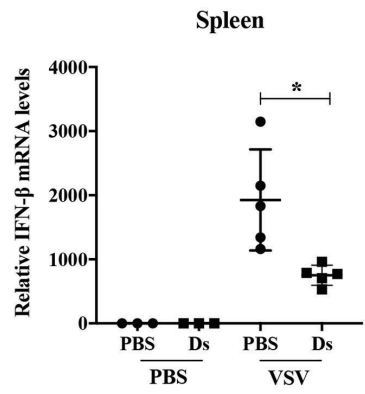

D

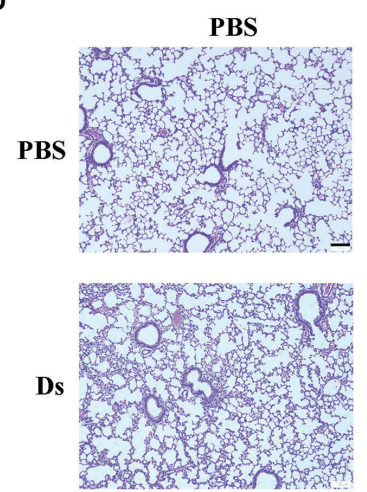

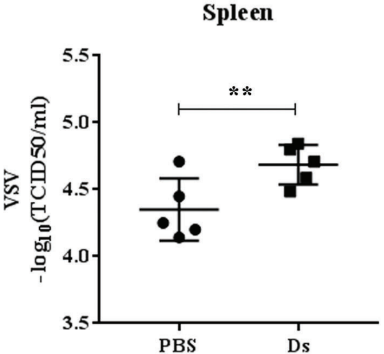

E
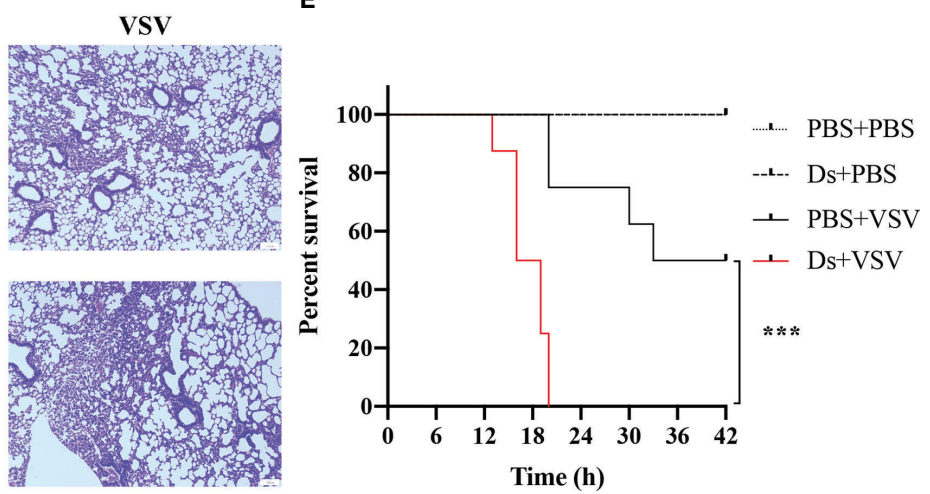

FIGURE 4 | Succinate suppresses the antiviral response of mice in vivo. (A-D) Mice $(6$ weeks old $)$ were infected with or without VSV $\left(1 \times 10^{8}\right.$ PFU/g) via intraperitoneal injection for $12 \mathrm{~h}$ after Ds or PBS treatment for $3 \mathrm{~h}$. VSV-non-infected group, $n=3 /$ group; VSV-infected groups, $n=5 /$ group. (A) The concentrations of IFN- $\beta$ in serum from Ds or PBS-pretreated mice were detected by ELISA. (B) The IFN- $\beta$ mRNA expression of lungs and spleens were determined by RT-qPCR. (C) The VSV loads in lungs and spleens were detected by TCID50. (D) Histological analysis of the lung tissue from mice in (A), scale bar $=100 \mu \mathrm{m}$. Images are representative of three independent experiments. (E) Mice (6 weeks old, $n=8)$ pretreated with Ds or PBS for $3 \mathrm{~h}$ were injected with a half-lethal dose of VSV (1.3 $\times$ $10^{7} \mathrm{PFU} / \mathrm{g}$ ) or PBS by tail intravenous injection, and survival was recorded for $42 \mathrm{~h}$. Log-rank test, ${ }^{\star \star *} p<0.001$. (A-C) Data are shown as mean \pm SD. Significance calculated using the unpaired $t$-test. ${ }^{*} p<0.05,{ }^{\star *} p<0.01$. Representative data are from three independent experiments.

\section{Succinate Inhibits VSV-Triggered MAVS-IRF3 Signaling}

To explore the mechanisms on which succinate regulated the antiviral response, we examined the MAVS-IRF3 signaling which triggers antiviral response by sequentially activating the downstream axis for type I IFN production. The RAW264.7 cells and MPMs were pretreated with $5 \mathrm{mM}$ Ds or DMSO and then infected with VSV $(\mathrm{MOI}=1)$. The cells were lysed at $0,2,4$, and $8 \mathrm{~h}$ post VSV infection, and the cell lysates were analyzed by Western blotting. As shown in Figure 5A, the MAVS, TBK1, and IRF3 phosphorylation was decreased in the Ds-pretreatment cells. Especially, at $8 \mathrm{~h}$ post-
VSV infection, the MAVS expression in the Ds-pretreatment RAW264.7 cells was nearly 0.4 -fold less than that in control cells. In the MPM cells, the MAVS, TBK1, and IRF3 phosphorylation was also decreased in the Ds-pretreatment cells (Figure 5B). Moreover, the MAVS expression in the Ds-pretreatment MPM cells was 0.8fold less than that in DMSO-pretreatment cells. The PBS-treated cells were used as normal control. The expression levels of MAVS, TBK1, and IRF3 phosphorylation did not change in the PBS control group between the DMSO and Ds-pretreatment cells (Figure S1). Together, the succinate inhibits VSV-triggered activation of MAVSIRF3 signaling. 
A RAW264.7
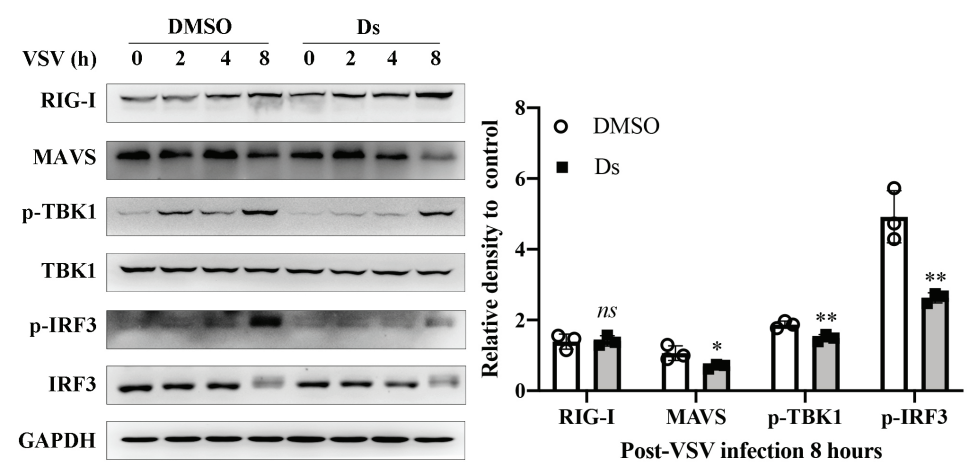

B
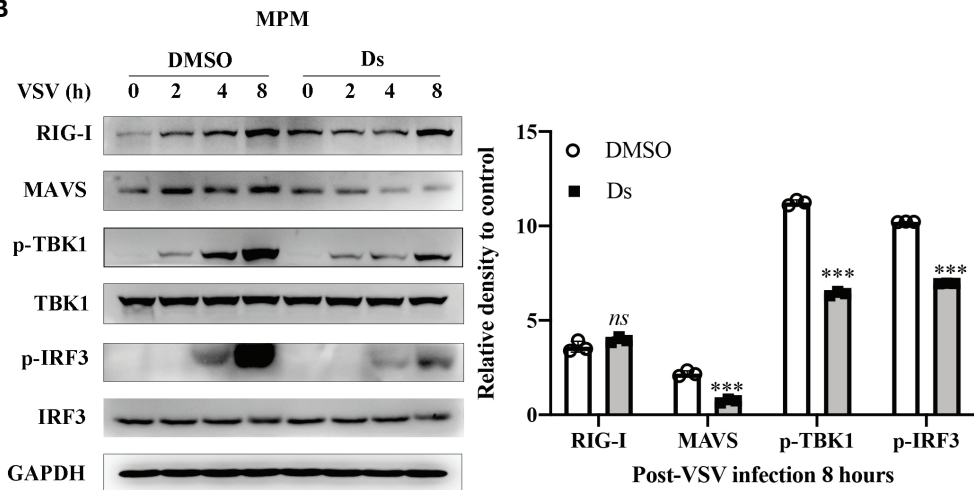

FIGURE 5 | Succinate inhibits VSV-triggered MAVS-IRF3 signaling. (A) RAW264.7 cells were pretreated with $5 \mathrm{mM}$ Ds or DMSO for $3 \mathrm{~h}$ followed infection by VSV $(\mathrm{MOI}=1)$ for the indicated times. Cell lysates were immunoblotted with the indicated antibodies. The histogram exhibited the densitometric measurement of RIG-I/ GAPDH, MAVS/GAPDH, p-IRF3/RF3, and p-TBK1/TBK1 ratio relative to $0 \mathrm{~h}$ post VSV infection. (B) MPM were pretreated with $5 \mathrm{mM}$ Ds or DMSO for $3 \mathrm{~h}$ followed infection by VSV $(\mathrm{MOI}=1)$ for the indicated times. Cell lysates were immunoblotted with the indicated antibodies. Cell lysates were immunoblotted with the indicated antibodies. The histogram exhibited the densitometric measuring of RIG-I/GAPDH, MAVS/GAPDH, p-IRF3/IRF3, and p-TBK1/TBK1 ratio relative to 0 h post VSV infection. Data are shown as mean \pm SD. Significance calculated using the unpaired $t$-test. ${ }^{*} p<0.05,{ }^{* *} p<0.01,{ }^{\star \star *} p<0.001$. Representative images are from three independent experiments.

\section{Succinate Suppresses the Activation and Aggregation of MAVS}

To further identify the molecular target of succinate in the VSVtriggered innate immune response, dual-luciferase reporter assay was performed using IFN- $\beta$ or ISRE luciferase reporter plasmid, phRL-TK plasmid, and RIG-I (N), MAVS, IRF3-5D, or TBK1 plasmids. At $24 \mathrm{~h}$ after transfection, IFN- $\beta$ (a) and ISRE (b) promoter activities were analyzed using the luciferase reporter to detect the target of succinate in the RIG-I-MAVS signaling pathway. The data revealed that only the RIG-I-N- and MAVStriggered IFN- $\beta$ and ISRE luciferase activation could be inhibited by the pretreatment of succinate (Figures 6A, B). These results suggested that MAVS might be the target molecule of succinate in the RIG-I-MAVS signaling pathway. It was reported that viral infection could induce the formation of very large MAVS aggregates, which potently activate IRF3 in the cytosol $(11,18)$; thus, we investigated whether succinate could reduce the aggregation levels of MAVS induced by VSV infection. The Flag-MAVS plasmids were transfected into HEK293T cells. At 3 h post Ds or DMSO pretreatment, the cells were infected with
VSV for $8 \mathrm{~h}$, and the cell lysates were loaded onto SDD-AGE to analyze the aggregation of MAVS. The results showed that the aggregation of MAVS was decreased in the Ds-pretreated cells (Figure 6C). Similarly, in the Ds-pretreated MPM cells, VSV infection induces lower aggregation of MAVS than that in the DMSO-pretreated cells (Figure 6D). Collectively, succinate partially inhibits the activation and aggregation of MAVS.

\section{DISCUSSION}

In the present study, upon VSV infection, the genes of glucose transporter (GLUT) 1 and GLUT4 were increased, and isocitrate dehydrogenase 1 (IDH1) mRNA and $\alpha$-ketoglutarate dehydrogenase $(\mathrm{OGDH})$ mRNA production was decreased in mouse macrophages, indicating that VSV challenge leads to the glucose metabolism reprogramming. This finding is consistent with the recent studies showing that the changes in glucose metabolic pathways during RLR-induced type I IFN production are critical for host antiviral immune responses (15, 19, 20). 

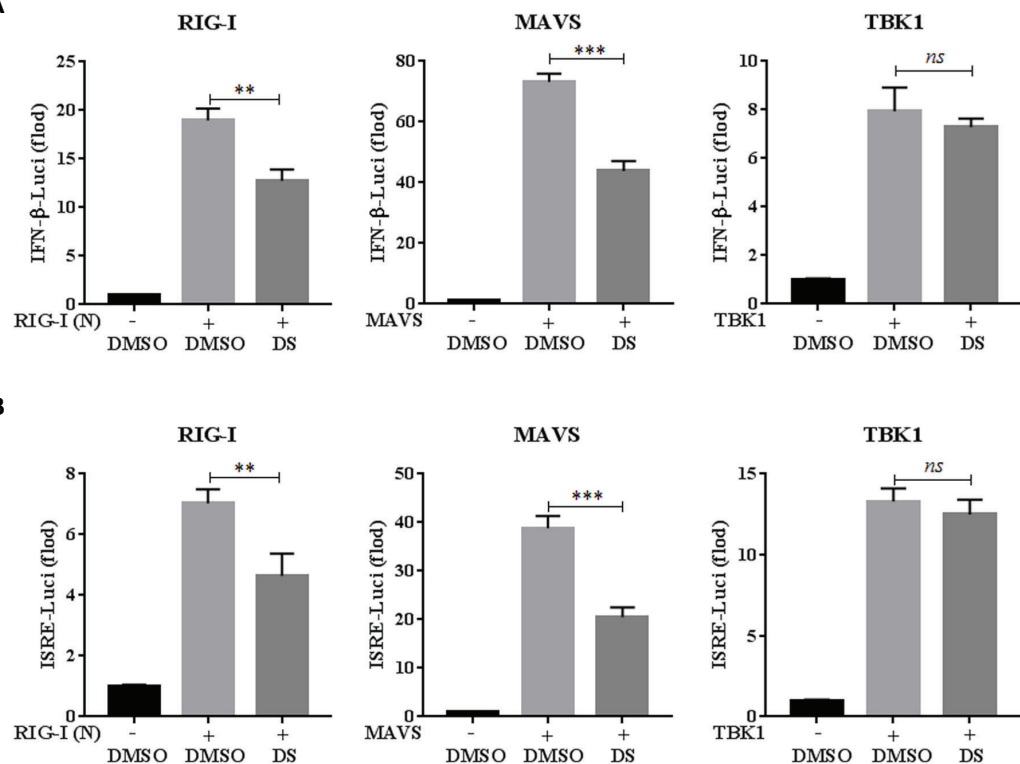

C

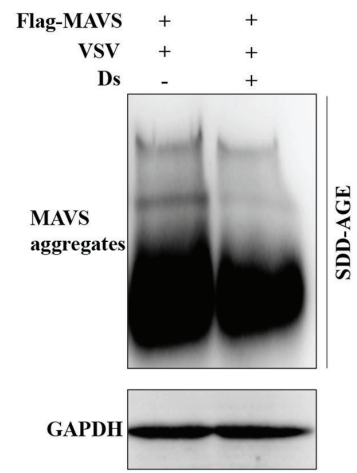

MAVS

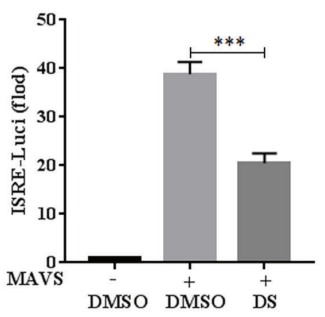

D

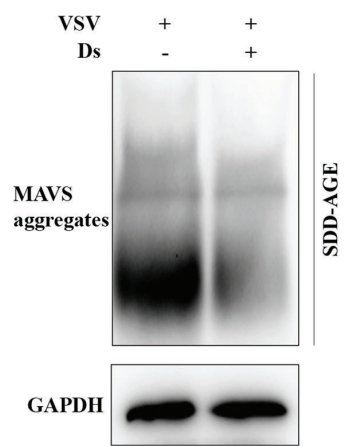

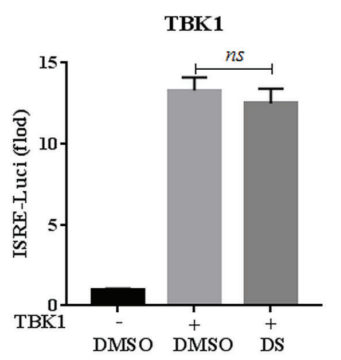

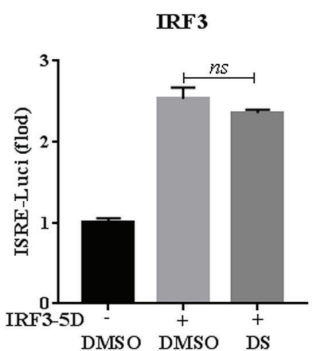

IRF3

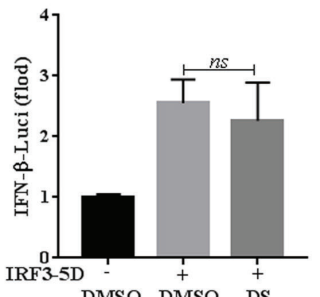

DMSO DMSO DS

FIGURE 6 | Succinate suppresses the activation and aggregation of MAVS. (A, B) 293T cells were pretreated with $5 \mathrm{mM}$ Ds or DMSO for $3 \mathrm{~h}$ and then cotransfected with the IFN- $\beta$ or ISRE luciferase reporter plasmid (90 ng), phRL-TK plasmid (10 ng), together with RIG-I (N), MAVS, IRF3-5D, or TBK1 plasmids (50 ng each). At $24 \mathrm{~h}$ after transfection, luciferase reporter assay was used to analysis of IFN- $\beta$ (A) and ISRE (B) promoter activity. Data are shown as mean \pm SD. Significance calculated using the unpaired $t$-test. ${ }^{* *} p<0.01,{ }^{* * *} p<0.001, n s$, no significant. (C) The HEK293T cells transfected with Flag-tagged MAVS plasmid were pretreated with Ds $(5 \mathrm{mM})$ or DMSO for $3 \mathrm{~h}$ and then infected with VSV for $8 \mathrm{~h}$. The cell lysates were prepared and analyzed by SDD-AGE using a MAVS antibody or SDS-PAGE using a GAPDH antibody. (D) The MPM cells were pretreated with Ds (5 mM) or DMSO for $3 \mathrm{~h}$ and then infected with VSV for $8 \mathrm{~h}$. The cell lysates were prepared and analyzed by SDD-AGE using a MAVS antibody or SDS-PAGE using a GAPDH antibody.

Especially, our results showed that the tricarboxylic acid (TCA) cycle metabolite succinate was gradually decreased with the prolongation of VSV infection time in RAW264.7 cells. The phenomenon of decreased succinate was also shown in HEK293T cells transfected with poly(I:C), following the activation of RLR signaling (15). In contrast, succinate was shown to accumulate in the lipopolysaccharide (LPS)-stimulated macrophages during inflammation and in the Salmonella typhimurium-infected macrophages during intracellular infection $(7,21)$. Therefore, succinate plays diverse and important roles as an immunometabolite in innate immune response including antiinflammatory response and antiviral response.

Our discovery that cell-permeable diethyl succinate inhibited the VSV-induced IFN- $\beta$ production in vitro and in vivo particularly accelerated the death of the VSV-infected mice, indicating a negative regulatory function of succinate in innate antiviral response. This study reveals for the first time the role of succinate in cell and host immune response to viral infection, even though substantial evidence accumulated in recent years has highlighted the role of succinate in regulating inflammatory response induced by multiple stimuli. Interestingly, it remains controversial whether succinate can be considered as a harmful signal or a protective molecule in the inflammatory response. In macrophages exposed to LPS, in a mouse model of antigeninduced arthritis, and in a mouse model of TNBS colitis, succinate showed a role of harmful signal to increase the production of pro-inflammatory cytokines $(4,6,7)$. However, there were some reports that showed that succinate acted as a 
protective molecule under different inflammatory conditions. Peruzzotti-Jametti et al. demonstrated that succinate activated calcium signaling and mitogen-activated protein kinase phosphorylation in rodent and human induced neural stem cells (iNSCs) and NSCs via mediating the activation of its receptor SUCNR1, and then elicited the anti-inflammatory function of iNSCs and NSCs by inducing the production of prostaglandin (PG) E2 (22). Moreover, the succinate-SUCNR1 signaling acted as a major driver of microbiota-triggered type 2 immunity in the intestine and was described as an antiinflammatory mediator to regulate the metabolic response to obesity in macrophages $(5,23)$. So far, most studies on succinate in inflammatory response and other physiologic and pathological circumstances including in the muscle in response to exercise training and in the tumor microenvironment have focused on its receptor SUCNR1 expressed on various cell surfaces $(24,25)$. Thus, during inflammation, whether succinate acts as a proinflammatory driver or an anti-inflammatory signal might be due to the status of immune cells expressing SUCNR1.

Without considering the function of SUCNR1 in antiviral response, we demonstrated that succinate could inhibit the aggregation of mitochondrial antiviral-signaling protein (MAVS) and inhibit its expression and activation, thereby negatively regulating RIG-I-MAVS signaling and leading to decreased IFN- $\beta$ production in VSV-infected mouse macrophages. In non-activated cells, MAVS is mainly as a monomer on the outer mitochondrial membrane in its monomeric form $(11,26,27)$. Upon engagement of viral RNA, RIG-I and MAVS proteins undergo conformational changes leading to the activation of antiviral signaling (28). It was noteworthy that MAVS forms well-ordered, functional prionlike aggregates, which potently activate IRF3 in the cytosol (11). In this study, we found that succinate could suppress the formation of MAVS aggregates and then decrease the phosphorylation levels of IRF3. In recent years, increasing evidence showed that many molecules regulated antiviral immune response by mediating the formation of MAVS aggregates. For example, the mitochondrial ubiquitin ligase MARCH5 was reported to resolves MAVS aggregates during antiviral signaling (29). Besides, RNF34 promoted the autophagic degradation of MAVS aggregates to negatively regulate the innate immune response (30). Moreover, SARSCoV-2 membrane glycoprotein $\mathrm{M}$ antagonizes the antiviral signaling by impairing MAVS aggregation and its recruitment of downstream TBK1 and IRF3 (31). Notably, glycolysis-derived lactate serves as a key metabolite and was shown to negatively regulate the RLR signaling by directly binding to the MAVS transmembrane domain and preventing MAVS aggregation (15).

\section{REFERENCES}

1. Stienstra R, Netea-Maier RT, Riksen NP, Joosten LAB, Netea MG. Specific and Complex Reprogramming of Cellular Metabolism in Myeloid Cells During Innate Immune Responses. Cell Metab (2017) 26(1):142-56. doi: 10.1016/j.cmet.2017.06.001

2. Rubic T, Lametschwandtner G, Jost S, Hinteregger S, Kund J, Carballido-Perrig N, et al. Triggering the Succinate Receptor GPR91
It is therefore reasonable to assume that the MAVS protein was the target molecule of succinate in the antiviral signaling.

In summary, we verified that succinate could inhibit cellular and host innate antiviral response by targeting MAVS and inhibiting the formation of MAVS aggregates. Our study provides not only a cross-link between the crucial immunometabolite succinate and antiviral immune response but also offers a crucial paradigm and strategy for the management of viral infection.

\section{DATA AVAILABILITY STATEMENT}

The raw data supporting the conclusions of this article will be made available by the authors, without undue reservation.

\section{ETHICS STATEMENT}

The animal study was reviewed and approved by the National Institute of Health Guide for the Care and Use of Laboratory Animals with the approval of Shenzhen University (Approval Number: AEWC-20200316).

\section{AUTHOR CONTRIBUTIONS}

WC and YX conceived and designed the experiment. YX, XC, ZhuW, JQ, and HT performed the experiments. XZ, HW, QD, and ZhuW analyzed the data. WC and YX processed and typeset the figures. WC, YX, and ZheW wrote the manuscript. All authors contributed to the article and approved the submitted version.

\section{FUNDING}

This work was supported by grants from the National Natural Science Foundation of China (Nos. U1801283, 31870908), Shenzhen Science and Technology Innovation Commission grant (No. JCYJ20180507182253653, JCYJ20190808172201639), and SZU Top Ranking Project (No. 86000000210) to WC.

\section{SUPPLEMENTARY MATERIAL}

The Supplementary Material for this article can be found online at: https://www.frontiersin.org/articles/10.3389/fimmu.2022. 816378/full\#supplementary-material

on Dendritic Cells Enhances Immunity. Nat Immunol (2008) 9 (11):1261-9. doi: 10.1038/ni.1657

3. de Castro Fonseca M, Aguiar CJ, da Rocha Franco JA, Gingold RN, Leite MF. GPR91: Expanding the Frontiers of Krebs Cycle Intermediates. Cell Commun Signal (2016) 14:3. doi: 10.1186/s12964016-0126-1

4. Littlewood-Evans A, Sarret S, Apfel V, Loesle P, Dawson J, Zhang J, et al. GPR91 Senses Extracellular Succinate Released From Inflammatory 
Macrophages and Exacerbates Rheumatoid Arthritis. J Exp Med (2016) 213 (9):1655-62. doi: 10.1084/jem.20160061

5. Nadjsombati MS, McGinty JW, Lyons-Cohen MR, Jaffe JB, DiPeso L, Schneider C, et al. Detection of Succinate by Intestinal Tuft Cells Triggers a Type 2 Innate Immune Circuit. Immunity (2018) 49(1):33-41.e37. doi: 10.1016/j.immuni.2018.06.016

6. Macias-Ceja DC, Ortiz-Masia D, Salvador P, Gisbert-Ferrandiz L, Hernandez C, Hausmann M, et al. Succinate Receptor Mediates Intestinal Inflammation and Fibrosis. Mucosal Immunol (2019) 12(1):178-87. doi: 10.1038/s41385018-0087-3

7. Tannahill GM, Curtis AM, Adamik J, Palsson-McDermott EM, McGettrick AF, Goel G, et al. Succinate Is an Inflammatory Signal That Induces IL-1beta Through HIF-1alpha. Nature (2013) 496(7444):238-42. doi: 10.1038/ nature11986

8. Ivashkiv LB, Donlin LT. Regulation of Type I Interferon Responses. Nat Rev Immunol (2014) 14(1):36-49. doi: 10.1038/nri3581

9. Schlee M, Hartmann G. Discriminating Self From Non-Self in Nucleic Acid Sensing. Nat Rev Immunol (2016) 16(9):566-80. doi: 10.1038/nri.2016.78

10. Honda K, Takaoka A, Taniguchi T. Type I Interferon [Corrected] Gene Induction by the Interferon Regulatory Factor Family of Transcription Factors. Immunity (2006) 25(3):349-60. doi: 10.1016/j.immuni.2006.08.009

11. Hou F, Sun L, Zheng H, Skaug B, Jiang QX, Chen ZJ. MAVS Forms Functional Prion-Like Aggregates to Activate and Propagate Antiviral Innate Immune Response. Cell (2011) 146(3):448-61. doi: 10.1016/j.cell.2011.06.041

12. Chen W, Han C, Xie B, Hu X, Yu Q, Shi L, et al. Induction of Siglec-G by RNA Viruses Inhibits the Innate Immune Response by Promoting RIG-I Degradation. Cell (2013) 152(3):467-78. doi: 10.1016/j.cell.2013.01.011

13. Feng H, Lenarcic EM, Yamane D, Wauthier E, Mo J, Guo H, et al. NLRX1 Promotes Immediate IRF1-Directed Antiviral Responses by Limiting dsRNAActivated Translational Inhibition Mediated by PKR. Nat Immunol (2017) 18 (12):1299-309. doi: 10.1038/ni.3853

14. Jin S, Tian S, Luo M, Xie W, Liu T, Duan T, et al. Tetherin Suppresses Type I Interferon Signaling by Targeting MAVS for NDP52-Mediated Selective Autophagic Degradation in Human Cells. Mol Cell (2017) 68(2):30822.e304. doi: 10.1016/j.molcel.2017.09.005

15. Zhang W, Wang G, Xu ZG, Tu H, Hu F, Dai J, et al. Lactate Is a Natural Suppressor of RLR Signaling by Targeting MAVS. Cell (2019) 178(1):17689.e115. doi: 10.1016/j.cell.2019.05.003

16. Hu J, Gao Q, Yang Y, Xia J, Zhang W, Chen Y, et al. Hexosamine Biosynthetic Pathway Promotes the Antiviral Activity of SAMHD1 by Enhancing OGlcNAc Transferase-Mediated Protein O-GlcNAcylation. Theranostics (2021) 11(2):805-23. doi: 10.7150/thno.50230

17. Li H, Zhao Z, Ling J, Pan L, Zhao X, Zhu H, et al. USP14 Promotes K63Linked RIG-I Deubiquitination and Suppresses Antiviral Immune Responses. Eur J Immunol (2019) 49(1):42-53. doi: 10.1002/eji.201847603

18. Shao WH, Shu DH, Zhen Y, Hilliard B, Priest SO, Cesaroni M, et al. PrionLike Aggregation of Mitochondrial Antiviral Signaling Protein in Lupus Patients Is Associated With Increased Levels of Type I Interferon. Arthritis Rheumatol (2016) 68(11):2697-707. doi: 10.1002/art.39733

19. Bajwa G, DeBerardinis RJ, Shao B, Hall B, Farrar JD, Gill MA. Cutting Edge: Critical Role of Glycolysis in Human Plasmacytoid Dendritic Cell Antiviral Responses. J Immunol (2016) 196(5):2004-9. doi: 10.4049/jimmunol.1501557

20. Li T, Li X, Attri KS, Liu C, Li L, Herring LE, et al. O-GlcNAc Transferase Links Glucose Metabolism to MAVS-Mediated Antiviral Innate Immunity. Cell Host Microbe (2018) 24(6):791-803.e796. doi: 10.1016/j.chom.2018.11.001
21. Rosenberg G, Yehezkel D, Hoffman D, Mattioli CC, Fremder M, Ben-Arosh $\mathrm{H}$, et al. Host Succinate Is an Activation Signal for Salmonella Virulence During Intracellular Infection. Science (2021) 371(6527):400-5. doi: 10.1126/ science.aba8026

22. Peruzzotti-Jametti L, Bernstock JD, Vicario N, Costa ASH, Kwok CK, Leonardi T, et al. Macrophage-Derived Extracellular Succinate Licenses Neural Stem Cells to Suppress Chronic Neuroinflammation. Cell Stem Cell (2018) 22(3):355-68.e313. doi: 10.1016/j.stem.2018.01.020

23. Keiran N, Ceperuelo-Mallafre V, Calvo E, Hernandez-Alvarez MI, Ejarque M, Nunez-Roa C, et al. SUCNR1 Controls an Anti-Inflammatory Program in Macrophages to Regulate the Metabolic Response to Obesity. Nat Immunol (2019) 20(5):581-92. doi: 10.1038/s41590-019-0372-7

24. Starling S. Succinate Regulates Muscle Exercise Adaptations. Nat Rev Endocrinol (2020) 16(12):678-9. doi: 10.1038/s41574-020-00429-2

25. Wu JY, Huang TW, Hsieh YT, Wang YF, Yen CC, Lee GL, et al. CancerDerived Succinate Promotes Macrophage Polarization and Cancer Metastasis via Succinate Receptor. Mol Cell (2020) 77(2):213-27.e215. doi: 10.1016/ j.molcel.2019.10.023

26. Baril M, Racine ME, Penin F, Lamarre D. MAVS Dimer Is a Crucial Signaling Component of Innate Immunity and the Target of Hepatitis C Virus NS3/4A Protease. J Virol (2009) 83(3):1299-311. doi: 10.1128/JVI.01659-08

27. Moresco EM, Vine DL, Beutler B. Prion-Like Behavior of MAVS in RIG-I Signaling. Cell Res (2011) 21(12):1643-5. doi: 10.1038/cr.2011.155

28. Peisley A, Wu B, Xu H, Chen ZJ, Hur S. Structural Basis for Ubiquitin-Mediated Antiviral Signal Activation by RIG-I. Nature (2014) 509(7498):110-4. doi: 10.1038 /nature 13140

29. Yoo YS, Park YY, Kim JH, Cho H, Kim SH, Lee HS, et al. The Mitochondrial Ubiquitin Ligase MARCH5 Resolves MAVS Aggregates During Antiviral Signalling. Nat Commun (2015) 6:7910. doi: 10.1038/ ncomms 8910

30. He X, Zhu Y, Zhang Y, Geng Y, Gong J, Geng J, et al. RNF34 Functions in Immunity and Selective Mitophagy by Targeting MAVS for Autophagic Degradation. EMBO J (2019) 38(14):e100978. doi: 10.15252/embj.2018100978

31. Fu YZ, Wang SY, Zheng ZQ, Yi H, Li WW, Xu ZS, et al. SARS-CoV-2 Membrane Glycoprotein M Antagonizes the MAVS-Mediated Innate Antiviral Response. Cell Mol Immunol (2021) 18(3):613-20. doi: 10.1038/ s41423-020-00571-x

Conflict of Interest: The authors declare that the research was conducted in the absence of any commercial or financial relationships that could be construed as a potential conflict of interest.

Publisher's Note: All claims expressed in this article are solely those of the authors and do not necessarily represent those of their affiliated organizations, or those of the publisher, the editors and the reviewers. Any product that may be evaluated in this article, or claim that may be made by its manufacturer, is not guaranteed or endorsed by the publisher.

Copyright $\odot 2022$ Xiao, Chen, Wang, Quan, Zhao, Tang, Wu, Di, Wu and Chen. This is an open-access article distributed under the terms of the Creative Commons Attribution License (CC BY). The use, distribution or reproduction in other forums is permitted, provided the original author(s) and the copyright owner(s) are credited and that the original publication in this journal is cited, in accordance with accepted academic practice. No use, distribution or reproduction is permitted which does not comply with these terms. 